\title{
Evidence on on-pump versus off-pump in Taiwan accepts a critical insight!
}

\author{
Paul Sergeant, MD, PhD, Prof em
}

\author{
From the Cardiovascular Surgery Department, Gasthuisberg University Hospital, Leuven, Belgium. \\ Disclosures: Author has nothing to disclose with regard to commercial support. \\ Received for publication Aug 12, 2018; accepted for publication Aug 17, 2018; available ahead of print Sept 22, \\ 2018 \\ Address for reprints: Paul Sergeant, MD, PhD, Prof em, Gasthuisberg University Hospital, Cardiovascular Sur- \\ gery Department, Herestraat, 3000 Leuven, Belgium (E-mail: paulsergeant133@gmail.com). \\ J Thorac Cardiovasc Surg 2019;157:974-5 \\ $0022-5223 / \$ 36.00$ \\ Copyright (c) 2018 by The American Association for Thoracic Surgery \\ https://doi.org/10.1016/j.jtcvs.2018.08.013
}

The research group of Chi and colleagues ${ }^{1}$ produced an interesting article concerning an on-pump versus offpump coronary artery bypass grafting (CABG) study of diabetic patients. The study evaluates the early risks and late benefits of a nationwide dataset, based on the National Health Insurance Research Database (NHIRD). Diabetes is a major health burden around the world, with more than 400 million patients with type 2 diabetes $^{2}$ estimated in 2015 , or $8 \%$ of the adult population. ${ }^{3}$

The major inferences from this study are that the offpump approach has reduced risk (mortality) in the very early phase and that no late differences were observed in late mortality $(P=.16)$ in a combined outcome of mortality, infarct, and stroke $(P=.82)$. This is after correcting for variability using a propensity-matched dataset of 7592 diabetic patients. This is not a randomized controlled trial, but its level of unfiltered evidence is high. The article by Chi and colleagues ${ }^{1}$ mandates a critical appraisal before it can be used in filtered systematic reviews.

The flowchart provided in Figure 1 of the article by Chi and colleagues ${ }^{1}$ starts with a national population of 41,155 patients undergoing CABG as an isolated or combined procedure between 2000 and 2011 in Taiwan and finally selects a population of 7592 diabetic patients after primary CABG, with 3796 in each cohort. Diabetic patients combines patients with oral therapy and patients with insulin-treated therapy. Only 990 patients had insulintreated diabetes, the group with the highest disease impact on morbidity and mortality.

Chi and colleagues ${ }^{1}$ tried to identify the off-pump versus the on-pump approach core variable. They decided to exclude the on-pump beating heart approach based on the consideration that this group would include the conversions from off-pump to on-pump. The study is not intention-totreat or start-to-treat and therefore might positively influence the off-pump approach by excluding the patients in whom the off-pump approach required a conversion. The on-pump approach is defined only as an arrested heart

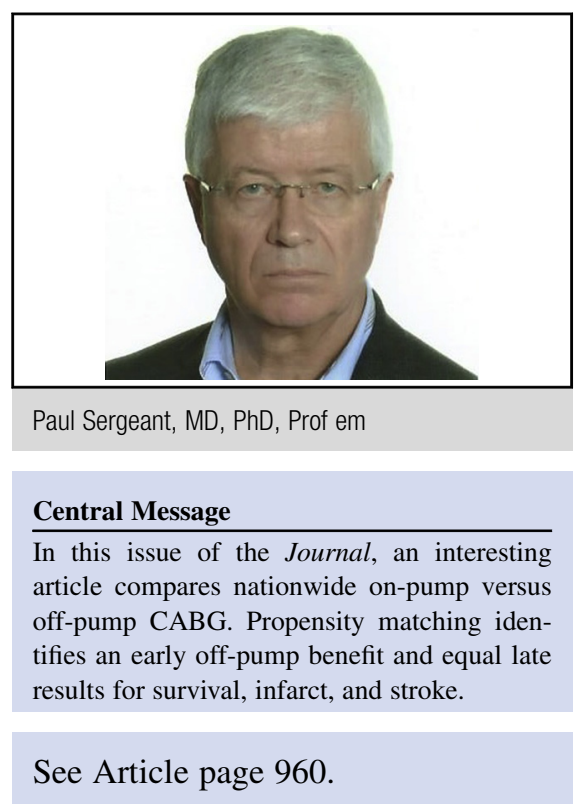

procedure supported by an extracorporeal circulation. This approach has only minor variability. The off-pump approach has not been defined except by the exclusion of any extracorporeal circulation, so there was no standardization on anesthesia, monitoring, anticoagulation, no-touch aorta, inotropes, enucleation methods, and shunting. These components have a major impact on the early hazard phase. This might negatively influence the off-pump approach.

The variables used in this study are those reported in the NHIRD, and all are categoric variables (even age and hospital volume). Matching cohorts with categoric variables is easy but not a fine correction. As an example, 2 patients can be classified as having chronic obstructive pulmonary disease, one with an $80 \% 1$-second value and the other with a 50\% 1-second value. They are unequal in risk, because risk is rarely in average values and most often in the outliers of risk. They also do not include variables known to influence the late repeat revascularization or survival of a patient who underwent $\mathrm{CABG}$, such as the completeness of arterial grafting or the no-touch aorta. A "rich" propensity model demands continuous variables to be able to discriminate between cohorts. This is why the C-stat of the propensity model cannot be classified as "saturated."

The outcome intervals are 30 days, 1 year, and late (defined as 10 years with an average of 5 years). Whenever 
a continuous event as death is defined by different predefined intervals, insight is lost and bias occurs. The 30-day interval is outdated when every hospital can place a patient on extracorporeal membrane oxygenation and dialysis beyond this interval. It would have been more interesting to have the data define the intervals and not have this predefined.

The outcome events are death, stroke, and infarct (independent or combined). It is unclear whether the variable definitions used by the NHIRD database are well defined and whether the reported data are audited or real clinical data. Death is probably a well-defined and audited event for such a national database. An independent outcome event is repeat revascularization, but because neither the completeness of revascularization nor the use of arterial grafting is reported or used in the propensity matching, this evidence becomes fragile.

The method used for correction of variability is propensity score matching. This is one of the 3 available propensity score possibilities. It is a powerful tool and widely accepted. Chi and colleagues ${ }^{1}$ include a variable called "year of surgery," which needs to be avoided in propensity scoring. The core quality criterion of a propensity model is the C-stat. It is called "saturated" and high quality if it reaches the 0.8 level, and below that level it is called "poor." This study has a C-stat of 0.782 . The inexperienced reader should not be misled. It is not easy to reach 0.78 to 0.8 with only categoric variables. This means that the correction for variability could have residual uncorrected variability between the 2 datasets.

Table 2 in the article by Chi and colleagues ${ }^{1}$ lists the observed number of events for the 3 predefined intervals. The differences in 30-day mortality immediately become evident, as well as the high 30-day mortality rates of $6.5 \%$ and $4.3 \%$. These high and recent (after 2000) rates create a major impact and are difficult to explain for this selected dataset of planned noncombined procedures. This high early mortality probably extends beyond the 30-day interval, but this is hidden in the interval between 30 days and 1 year. Table 2 lists the rates for 30 -day infarct as $0.21 \%$ and $0.26 \%$, but it is unclear how this variable has been defined and audited. If these rates are so low, why is the early mortality 20 times higher?

This increases even more the possible uncertainty around the variable stroke and if every neurologic dysfunction has been screened appropriately by similar neurologic expertise and imaging. Have the same definitions been used in follow-up as in the first 30 days for infarct and stroke because the medical professionals are probably different? This is not addressed.

The strengths and weakness of this article ${ }^{1}$ go in parallel. Using data from a functioning national database has major benefits, but we have to understand that this database was not designed as a clinical research database with hundreds of rich variables and that intentional or unintentional underreporting might have minimal, negative, or sometimes even positive financial effects in a database created for financial reporting. Chi and colleagues ${ }^{1}$ have written an interesting article. They have tried to resolve some issues as much as possible and have clearly outlined most of these limitations in a separate section. As usual, one should read the article before agreeing with the inferences.

\section{References}

1. Huang KC, Wu IH, Chou NK, Yang YY, Lin LC, Yu HY, et al. Late outcomes of offpump versus on-pump coronary bypass in diabetic patient: a nationwide study from Taiwan. J Thorac Cardiovasc Surg. 2019;157:960-9.

2. International Diabetes Foundation. IDF Diabetes Atlas. 7th ed. 2015. Available at: http://diabetesatlas.org/resources/2017-atlas.html. Accessed Sept 17, 2018

3. Shi Y, Hu FB. Global implication of diabetes and cancer. Lancet. 2014;383 1947-8. 